\title{
RNAi AND miRNA PATHWAYS IN FISH
}

Keywords: dsRNA, siRNA, miRNA, Dicer, TARBP2, PACT, Argonaute

\section{PETR SVOBODA}

Institute of Molecular Genetics, Academy of Sciences of the Czech Republic, Videnska 1083, 14220 Prague 4, Czech Republic

Correspondence to: Petr Svoboda, Institute of Molecular Genetics ASCR, Videnska 1083, 14220 Prague 4, Czech Republic, tel. \#+420 241063147, e-mail: svobodap@img.cas.cz.

\begin{abstract}
RNA silencing denotes sequence-specific repression mediated by small RNAs. In vertebrates, there are two closely related pathways, which share several protein factors: RNA interference (RNAi) and microRNA (miRNA) pathway. The miRNA pathway regulates endogenous protein-coding gene expression and has been implicated in many biological processes. RNAi generally serves as a form of innate immunity targeting viruses and mobile elements. This text reviews miRNA and RNAi pathways in fish. RNAi and other dsRNA-responding pathways in fish are very much like those in mammals, which is remarkable considering the mammalian and fish lineages separated in Paleozoicum some 400 million years ago. The miRNA pathway is likely the dominant small RNA pathway while, similarly to other vertebrates, the existence and functionality of endogenous RNAi remains unclear.
\end{abstract}

\section{Introduction}

Fish are an aquatic heterogeneous paraphyletic group with the majority of the species belonging to the bony fish class (Osteichthyes) group, which has 25000 species, the highest species diversity than any other vertebrate group (Margulis and Schwartz, 1998). Overall, the organization of small RNA pathways in fish is very similar to that of mammals (Fig. 1), which is notable considering the common ancestor of fish and mammals existed 3602-4500 million years ago (Volff, 2005).

Since mechanistical principles of vertebrate miRNA and RNAi pathways were introduced in the first two reviews of this series (Svoboda, 2019a, b) and in further detail elsewhere (Bartel, 2018; Svoboda, 2014), I will focus here directly on features of these pathways reported from fish models. Next generation sequencing analysis of zebrafish small RNAs identified miRNAs and germline piRNAs as common small RNAs. The dominant small RNA pathway in fish is the miRNA pathway. Studies of roles of miRNAs account for the vast majority of the literature on small RNA in fish. At the same time, the molecular 
mechanism of miRNA and RNAi pathways was seldom drectly studied in the fish model. The second most studied studied small RNA pathway in fish is the piRNA pathway, which protects the germline from mobile elements (Houwing et al., 2007; Huang et al., 2011; Kamminga et al., 2010) and differs from miRNA and RNAi pathways in small RNA biogenesis, which does not require Dicer.

\section{Dicer}

Fish genomes carry a single gene for Dicer, which is an ortholog of the mammalian Dicer and Dicer-1 in Drosophila (Murphy et al., 2008). This notion is supported by annotated fish genome data in the UCSC genome browser (https://genome.ucsc.edu/), and tblastn search of fish sequences at NCBI (e.g. Salmo salar, Danio rerio, Takifugu rubripes, Gasterosteus aculeatus (stickleback), Oryzias latipes (medaka)). Existence of a single Dicer gene in fish genomes is remarkable in teleost fish species, which underwent genome duplication (Howe et al., 2013; Meyer and Schartl, 1999). This suggests selective pressure could exist against Dicer gene duplication. Zebrafish Dicer is essential for development and its primary role seems to be miRNA biogenesis (Wienholds et al., 2003). The role of Dicer in endogenous RNAi in fish has not been addressed in much detail. A study of Dicer in grass carp Ctenopharyngodon idellar revealed a CDS encoding Dicer protein carrying all known functional domains found typically in other Dicers (Shen et al., 2013). Ctenopharyngodon idellar Dicer is abundantly expressed in brain, gill, head kidney, liver, spleen, heart, muscle and intestine. A positive correlation was found between Ctenopharyngodon idellar Dicer mRNA expression and infection with grass carp reovirus (GCRV) infection in cultured kidney cells and in the liver (8.46-fold, $\mathrm{P}<0.01,12 \mathrm{~h}$ post-infection) and spleen in vivo (Shen et al., 2013).This suggests that Ctenopharyngodon idellar Dicer is an inducible gene responding to viral infection although evidence for virus-derived endogenous siRNAs has not been provided.

\section{dsRBPs}

Fish genomes typically contain orthologs of TRBP2 and PACT, which are mammalian Dicer-interacting dsRBPs (Murphy et al., 2008). However, their function in small RNA silencing was not studied in the fish model, so far.

\section{Argonaute proteins}

Fish AGO proteins are orthologs of AGO proteins in other vertebrates (Murphy et al., 2008). However, teleost fish clade contains an additional AGO paralog, which emerged from a fish-specific genome duplication event that occurred approximately 350 million years ago (McFarlane et al., 2011). All five Ago genomic loci in teleosts contain specific, conserved sequence elements in non-coding regions indicating that the teleost AGO 
paralogs are differentially regulated, which is consistent with expression analysis in the zebrafish model. Multiple sequence alignments show that teleost homologs possess critical aminoacid residues for AGO function as well as ortholog-specific features retained throughout the vertebrate lineage (McFarlane et al., 2011).

\section{miRNA pathway}

In terms of small RNA research, fish models are typically used for exploring miRNA populations and studying biological roles of different miRNAs. Articles annotating miRNAs and analyzing their expression and function represent the bulk of the fish-related references. We have found 348 articles, which annotated and/or analyzed expression and function of miRNAs in fish. However, almost none of these articles brought any specific mechanistic insight into the molecular mechanism of miRNA in fish.

According to the miRBase (release 22.1), miRNA population in fish appears less complex than in mammals - fish models have less annotated miRNAs (Table 1) although there are dozens of reports on next generation sequencing analysis and miRNA annotation. However, zebrafish is an experimentally easily accessible model for exploring conserved roles of miRNAs in different tissues, which is also reflected in the number of references.

Table 1 Numbers of annotated miRNAs in selected fishes in miRBase 22.1

\begin{tabular}{|l|c|c|}
\hline species & miRNA precursors & mature miRNA \\
\hline Cyprinus carpio & 134 & 146 \\
\hline Danio rerio & 355 & 373 \\
\hline Fugu rubripes & 131 & 108 \\
\hline Ictalurus punctatus & 281 & 205 \\
\hline Oryzias latipes & 168 & 146 \\
\hline Salmo salar & 371 & 498 \\
\hline Tetraodon nigroviridis & 132 & 109 \\
\hline
\end{tabular}

In terms of the molecular mechanism of RNA silencing, studies in the zebrafish and other fish models brought several interesting discoveries concerning specific miRNA functions and unique adaptations in fish. Several examples have relevance for the research of the molecular mechanism of miRNA pathway:

The first example is the biology of the miR-430 family of miRNAs. While miRNAs in mice are essentially irrelevant for the oocyte-to-embryo transition (Suh et al., 2010), the zebrafish oocyte-to-embryo transition incorporates zygotically-expressed miR-430 family in maternal mRNA degradation (Giraldez et al., 2006; Mishima et al., 2006). Furthermore, the onset of miR-430 activity in the zebrafish zygote allowed for addressing the relationship between miRNA-induced translational repression and mRNA degradation (Bazzini et al., 2012; Mishima et al., 2012). Using ribosome profiling of zygotic stages, it was showed that miR-430 reduces translation before causing mRNA decay (Bazzini et al., 
2012). A significant technological outcome of these studies was development of target protectors, morpholino oligonucleotides specifically disrupting miRNA-mediated repression via hybridizing to and masking miRNA-binding sites (Choi et al., 2007).

Another contribution to small RNA biology coming from fish model was discovery of one of the non-canonical miRNA biogenesis mechanisms, namely Dicer-independent miRNA biogenesis of miR-451, which uses AGO2 slicing activity followed by uridylation and trimming (Cifuentes et al., 2010). The process also employs translation initiation factor eIF1A, which directly interacts with AGO2 and promotes miR-451 biogenesis (Yi et al., 2015).

Data from zebrafish also contributed to understanding other miRNA regulations. Two related terminal uridyl transferases (TUTases), Zcchc6 (TUT7) and Zcchc11 (TUT4), selectively 3 ' monouridylate a subset of miRNAs (Thornton et al., 2014). TUTase inhibition in zebrafish embryos causes developmental defects and aberrant Hox gene expression (Thornton et al., 2014).

Another miRNA regulator discovered in the zebrafish is dead end 1 (DND1), which is negatively regulating miRNA targeting. DND1 is an evolutionary conserved RNA-binding protein (RBP) that counteracts the function of several miRNAs in zebrafish primordial germ cells as well as in human cells. DND1 binds mRNAs and prohibits miRNAs from binding cognate mRNAs. DND1 effects involve uridine-rich regions present in the miRNA-targeted mRNAs (Kedde et al., 2007)

Taken together studies of molecular mechanism of miRNA-mediated repression in fish did not reveal any notable deviation from what has been observed in mammals. As the protein machinery appears to be well-conserved, miRNA pathways among vertebrate taxons primarily differ in sets of miRNAs and their targets, which dynamically evolve over time. This was for example demonstrated for zebrafish miR-430 and murine miR-290-295 miRNA clusters, which share common ancestry, both are associated with early development but do not regulate the same genes although some targets seem to be conserved (Svoboda and Flemr, 2010).

\section{RNAi}

The presence of RNAi response was examined in zebrafish at the same as in other animal models during the turn of the century. However, unlike specific RNAi observed in mouse oocytes and early embryos (Svoboda et al., 2000; Wianny and Zernicka-Goetz, 2000), long dsRNA injection into zebrafish had been yielding inconsistent results (Li et al., 2000; Mangos et al., 2001; Oates et al., 2000; Zhao et al., 2001). While some reported specific knockdown effects (Li et al., 2000; Mangos et al., 2001), others observed non-specific effects (Oates et al., 2000; Zhao et al., 2001). Non-specific effects remained a recurring theme also in later studies (Wang et al., 2010; Zhao et al., 2008) although some authors were able to achieve specific RNAi effects (De Rienzo et al., 2012; Dong et al., 2013; Ying et al., 2010).Non-specific effects in zebrafish embryos were not remedied by the use of siRNAs and it was later shown that the basis of the non-specific effects is interference with miRNA function (Zhao et al., 2008). Injection of zebrafish zygotes with siRNA caused a significant reduction in miR-430 levels leading to unspecific developmental defects (Zhao et al., 2008). 
Interestingly, literature survey revealed that experiments with the parasitic sea lamprey (Petromyzon marinus), a jawless fish relative, showed that uptake of free siRNA at $5 \mu \mathrm{g} / \mathrm{ml}$ did not trigger an RNAi response (Heath et al., 2014). In any case, RNAi did not become a favourite knock-down strategy to study genes during zebrafish development; microinjection of morpholino oligonucleotides (Blum et al., 2015; Eisen and Smith, 2008) became the preferred approach instead.

Taken together, successful RNAi experiments with long dsRNA demonstrate that zebrafish holds the molecular machinery for executing RNAi: Dicer, TARBP2, and AGO2. However, its capacity for mediating specific knock-down effects is limited because the same machinery is being simultaneously utilized by the miRNA pathway. Importantly, the availability of the machinery above the minimum capacity sustaining the miRNA pathway functionality likely differs during development and among different cell types.

While zebrafish holds the molecular machinery for executing RNAi, the question remains whether the endogenous RNAi has any significant role in fish. Available data do not provide unequivocal evidence for significant endogenous RNAi in fish. Next generation sequencing of small RNAs contains fractions of non-miRNA small RNAs of endo-siRNA size, yet it is not clear if these fragments truly represent bona fide endo-siRNAs. Some other data indirectly point to a possible antiviral role, namely GCRV-induced transient upregulation of Ago2 in rare minnow (Gobiocypris rarus) and Dicer upregulation in grass carp (Ctenopharyngodon idella) (Guo et al., 2012; Shen et al., 2013). Interaction of GCRV with the small RNA machinery (the miRNA pathway should not be excluded) has been suggested based on the observation in grass carp kidney cells that GCRV dsRNA could be processed into siRNAs but GCRV infection did not yield GCRV-derived siRNAs while Dicer upregulation occurred (Gotesman et al., 2014). It has been thus proposed that an unidentified RNAi suppressor might contribute to the survival of the viral genome and efficient viral replication (Gotesman et al., 2014). The presence of a virus-derived inhibitor of RNA silencing in a fish RNA virus would be indicative of an existing antiviral role of small RNAs, which is being suppressed. However, an alternative scenario that should be considered as well is that dsRNA formed during viral replication is not accessible for Dicer-mediated cleavage. Therefore, further research is needed to address this issue.

\section{Other notable silencing phenomena}

There are two phenomena, which clearly overlap with RNA silencing but their underlying molecular mechanism remains unclear and will require further investigation.

Andrews et al found that introduction of transgenes containing convergent transcription units in zebrafish embryos induced stable transcriptional gene silencing in cis and trans. The silencing was suppressed upon Dicer knockdown, indicating processing of double stranded RNA. ChIP revealed that silencing was accompanied by enrichment of the constitutive heterochromatin mark H3K9me3 (Andrews et al., 2014). While small RNA-induced transcriptional silencing is well established in fungi and plants (and seems to be a product of convergent evolution), the molecular mechanism underlying seemingly related observations in vertebrates is unclear. 
The second phenomenon deals with the role of small RNAs during DNA-damage response, which has been discussed above in the mammalian section (Francia et al., 2012). It was shown in zebrafish that DICER and DROSHA, but not downstream elements of the RNAi pathway, are necessary to activate the DDR upon exogenous DNA damage and oncogene-induced genotoxic stress, as studied by DDR foci formation and by checkpoint assays. It was also reported that formation of DDR foci requires site-specific DICER- and DROSHA-dependent small RNAs, which are sufficient to restore the DDR in RNase-Atreated cells (Francia et al., 2012).

\section{Summary}

The molecular mechanism of RNAi and miRNA pathways in fish seems to be essentially identical to that of mammals (Fig. 1). While there are two dsRBPs, the role of PACT in RNA silencing remains to be determined. miRNA pathway is the dominant small RNA pathway while the existence and functionality of endogenous RNAi are unclear. Some variations (DNA-binding PKR homologs (Rothenburg et al., 2008)) were found in the interferon system, which is the main fish antiviral system.
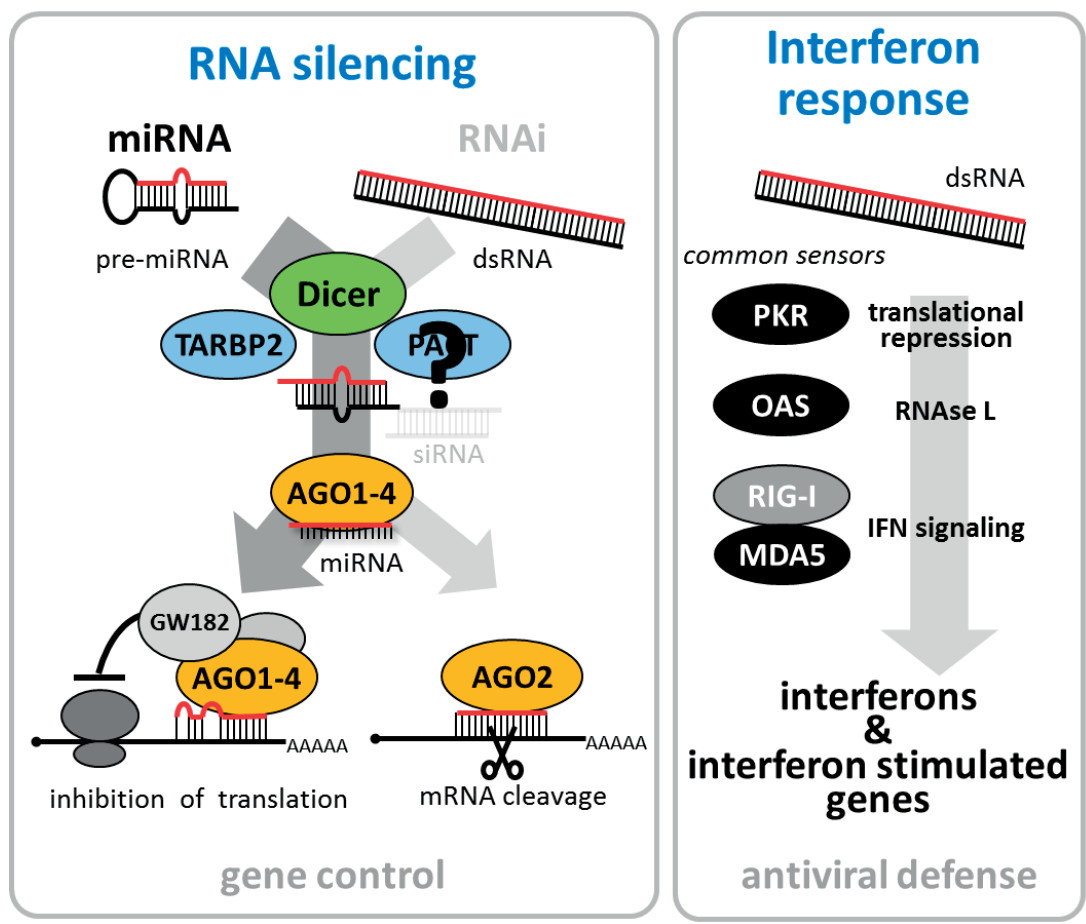

Figure 1 Overview of RNA silencing and dsRNA response in FISH dsRNA and miRNA pathways in bony fishes are very much similar to the mammalian ones. 


\section{Acknowledgement}

I would like to thank my colleagues Jan Paces, Miloslav Nic, and Tomas Novotny for help with collecting literature for the review. The review content was produced under a contract OC/EFSA/GMO/2015/01-CT 01 with European Food Safety Authority (EFSA); the opinions expressed are those of the contractor only and do not represent EFSA's official position. Publication of the review was funded by LO1220 and LM2015063 by the Ministry of Education, Youth and Sports.

\section{References}

Andrews, O.E., Cha, D.J., Wei, C.Y., and Patton, J.G. (2014). RNAi-Mediated Gene silencing in Zebrafish Triggered by Convergent Transcription. Scientific Reports 4, 5222-5222.

Bartel, D.P. (2018). Metazoan MicroRNAs. Cell 173, 20-51.

Bazzini, A.A., Lee, M.T., and Giraldez, A.J. (2012). Ribosome Profiling Shows That miR-430 Reduces Translation Before Causing mRNA Decay in Zebrafish. Science 336, 233-237.

Blum, M., De Robertis, E.M., Wallingford, J.B., and Niehrs, C. (2015). Morpholinos: Antisense and Sensibility. Developmental Cell 35, 145-149.

Choi, W.Y., Giraldez, A.J., and Schier, A.F. (2007). Target protectors reveal dampening and balancing of nodal agonist and antagonist by miR-430. Science 318, 271-274.

Cifuentes, D., Xue, H., Taylor, D.W., Patnode, H., Mishima, Y., Cheloufi, S., Ma, E., Mane, S., Hannon, G.J., Lawson, N.D., et al. (2010). A novel miRNA processing pathway independent of dicer requires argonaute2 catalytic activity. Science 328, 1694-1698.

De Rienzo, G., Gutzman, J.H., and Sive, H. (2012). Efficient shRNA-Mediated Inhibition of Gene Expression in Zebrafish. Zebrafish 9, 97-107.

Dong, Z.Q., Peng, J.S., and Guo, S. (2013). Stable Gene Silencing in Zebrafish with Spatiotemporally Targetable RNA Interference. Genetics 193, 1065-+.

Eisen, J.S., and Smith, J.C. (2008). Controlling morpholino experiments: don’t stop making antisense. Development 135, 1735-1743.

Francia, S., Michelini, F., Saxena, A., Tang, D., de Hoon, M., Anelli, V., Mione, M., Carninci, P., and d'Adda di Fagagna, F. (2012). Site-specific DICER and DROSHA RNA products control the DNA-damage response. Nature 488, 231-235.

Giraldez, A.J., Mishima, Y., Rihel, J., Grocock, R.J., Van Dongen, S., Inoue, K., Enright, A.J., and Schier, A.F. (2006). Zebrafish MiR-430 promotes deadenylation and clearance of maternal mRNAs. Science 312, 75-79.

Gotesman, M., Soliman, H., Besch, R., and El-Matbouli, M. (2014). In vitro inhibition of Cyprinid herpesvirus-3 replication by RNAi. J Virol Methods 206, 63-66.

Guo, S., Xu, D., Xu, H.-X., Wang, T., Li, J.-1., and Lu, L.-q. (2012). Suppression of RNA interference pathway in vitro by Grass carp reovirus. Virologica Sinica 27, 109-119.

Heath, G., Childs, D., Docker, M.F., McCauley, D.W., and Whyard, S. (2014). RNA Interference Technology to Control Pest Sea Lampreys - A Proof-of-Concept. Plos One 9, e88387-e88387. 
Houwing, S., Kamminga, L.M., Berezikov, E., Cronembold, D., Girard, A., van den Elst, H., Filippov, D.V., Blaser, H., Raz, E., Moens, C.B., et al. (2007). A role for Piwi and piRNAs in germ cell maintenance and transposon silencing in zebrafish. Cell 129, 69-82.

Howe, K., Clark, M.D., Torroja, C.F., Torrance, J., Berthelot, C., Muffato, M., Collins, J.E., Humphray, S., McLaren, K., Matthews, L., et al. (2013). The zebrafish reference genome sequence and its relationship to the human genome. Nature 496, 498-503.

Huang, H.Y., Houwing, S., Kaaij, L.J.T., Meppelink, A., Redl, S., Gauci, S., Vos, H., Draper, B.W., Moens, C.B., Burgering, B.M., et al. (2011). Tdrd1 acts as a molecular scaffold for Piwi proteins and piRNA targets in zebrafish. EMBO J 30, 3298-3308.

Kamminga, L.M., Luteijn, M.J., den Broeder, M.J., Redl, S., Kaaij, L.J.T., Roovers, E.F., Ladurner, P., Berezikov, E., and Ketting, R.F. (2010). Hen1 is required for oocyte development and piRNA stability in zebrafish. EMBO J 29, 3688-3700.

Kedde, M., Strasser, M.J., Boldajipour, B., Vrielink, J., Le Sage, C., Nagel, R., Voorhoeve, P.M., Van Duijse, J., Orom, U.A., Lund, A.H., et al. (2007). RNA-binding protein Dnd1 inhibits microRNA access to target mRNA. Cell 131, 1273-1286.

Li, Y.X., Farrell, M.J., Liu, R.P., Mohanty, N., and Kirby, M.L. (2000). Double-stranded RNA injection produces null phenotypes in zebrafish. Developmental Biology 217, 394-405.

Mangos, S., Vanderbeld, B., Krawetz, R., Sudol, K., and Kelly, G.M. (2001). Ran binding protein RanBP1 in zebrafish embryonic development. Molecular Reproduction and Development 59, 235-248.

Margulis, L., and Schwartz, K.V. (1998). Five kingdoms : an illustrated guide to the phyla of life on earth, 3rd edn (New York: W.H. Freeman).

McFarlane, L., Svingen, T., Braasch, I., Koopman, P., Schartl, M., and Wilhelm, D. (2011). Expansion of the Ago gene family in the teleost clade. Development Genes and Evolution 221, 95-104.

Meyer, A., and Schartl, M. (1999). Gene and genome duplications in vertebrates: the one-tofour (-to-eight in fish) rule and the evolution of novel gene functions. Curr Opin Cell Biol 11, 699-704.

Mishima, Y., Fukao, A., Kishimoto, T., Sakamoto, H., Fujiwara, T., and Inoue, K. (2012). Translational inhibition by deadenylation-independent mechanisms is central to microRNA-mediated silencing in zebrafish. Proc Natl Acad Sci U S A 109, 1104-1109.

Mishima, Y., Giraldez, A.J., Takeda, Y., Fujiwara, T., Sakamoto, H., Schier, A.F., and Inoue, K. (2006). Differential regulation of germline mRNAs in soma and germ cells by zebrafish miR-430. Current Biology 16, 2135-2142.

Murphy, D., Dancis, B., and Brown, J.R. (2008). The evolution of core proteins involved in microRNA biogenesis. BMC Evolutionary Biology 8, 92-92.

Oates, A.C., Bruce, A.E.E., and Ho, R.K. (2000). Too much interference: Injection of double-stranded RNA has nonspecific effects in the zebrafish embryo. Developmental Biology 224, 20-28.

Rothenburg, S., Deigendesch, N., Dey, M., Dever, T.E., and Tazi, L. (2008). Double-stranded RNA-activated protein kinase PKR of fishes and amphibians: Varying the number of double-stranded RNA binding domains and lineage-specific duplications. BMC Biology 6, 12-12.

Shen, X.B., Xu, D., Li, J.L., and Lu, L.Q. (2013). Molecular cloning and immune responsive expression of a ribonuclease III orthologue involved in RNA interference, dicer, in grass carp Ctenopharyngodon idella. Journal of Fish Biology 83, 1234-1248. 
Suh, N., Baehner, L., Moltzahn, F., Melton, C., Shenoy, A., Chen, J., and Blelloch, R. (2010). MicroRNA Function Is Globally Suppressed in Mouse Oocytes and Early Embryos. Current Biology 20, 271-277.

Svoboda, P. (2014). Renaissance of mammalian endogenous RNAi. FEBS Lett 588, 2550-2556.

Svoboda, P. (2019a). Introduction to RNAi and miRNA pathways.

Svoboda, P. (2019b). RNAi and miRNA pathways in mammals I - molecular mechanisms.

Svoboda, P., and Flemr, M. (2010). The role of miRNAs and endogenous siRNAs in maternal-to-zygotic reprogramming and the establishment of pluripotency. EMBO reports 11, 590-597.

Svoboda, P., Stein, P., Hayashi, H., and Schultz, R.M. (2000). Selective reduction of dormant maternal mRNAs in mouse oocytes by RNA interference. Development 127, 4147-4156.

Thornton, J.E., Du, P., Jing, L.L., Sjekloca, L., Lin, S.B., Grossi, E., Sliz, P., Zon, L.I., and Gregory, R.I. (2014). Selective microRNA uridylation by Zcchc6 (TUT7) and Zcchc11 (TUT4). Nucleic Acids Research 42, 11777-11791.

Volff, J.N. (2005). Genome evolution and biodiversity in teleost fish. Heredity (Edinb) 94, 280-294.

Wang, L., Zhou, J.Y., Yao, J.H., Lu, D.R., Qiao, X.J., and Jia, W. (2010). U6 promoter-driven siRNA injection has nonspecific effects in zebrafish. Biochem Biophys Res Commun 391, 1363-1368.

Wianny, F., and Zernicka-Goetz, M. (2000). Specific interference with gene function by double-stranded RNA in early mouse development. Nature Cell Biology 2, 70-75.

Wienholds, E., Koudijs, M.J., van Eeden, F.J.M., Cuppen, E., and Plasterk, R.H.A. (2003). The microRNA-producing enzyme Dicer1 is essential for zebrafish development. Nature Genetics 35, $217-218$.

Yi, T.F., Arthanari, H., Akabayov, B., Song, H.D., Papadopoulos, E., Qi, H.H., Jedrychowski, M., Guttler, T., Guo, C.C., Luna, R.E., et al. (2015). eIF1A augments Ago2-mediated Dicer-independent miRNA biogenesis and RNA interference. Nature Communications 6, 7194-7194.

Ying, S.Y., Chang, C.P., and Lin, S.L. (2010). Intron-Mediated RNA Interference, Intronic MicroRNAs, and Applications. In Rna Therapeutics: Function, Design, and Delivery, pp. 203-235.

Zhao, X.F., Fjose, A., Larsen, N., Helvik, J.V., and Drivenes, O. (2008). Treatment with small interfering RNA affects the microRNA pathway and causes unspecific defects in zebrafish embryos. FEBS Journal 275, 2177-2184.

Zhao, Z.X., Cao, Y., Li, M., and Meng, A.M. (2001). Double-stranded RNA injection produces nonspecific defects in zebrafish. Developmental Biology 229, 215-223. 